\title{
LAS NODRIZAS DE LAS INCLUSAS. LAS AMAS DE LECHE DE LA CASA DE MATERNIDAD Y EXPÓSITOS DE BARCELONA, 1853-1903
}

\author{
Ana María Rodríguez Martín \\ Catedrática de AFA El Clot \\ anarmartin@yahoo.es
}

Recibido: 28-02-09

Aceptado: 06-03-09

\section{Resumen}

Este artículo analiza las características de las nodrizas de las inclusas, la consideración social de estas mujeres, su influencia sobre la mortalidad de los expósitos y su papel dentro de estas instituciones benéficas y de la Casa de Maternidad y Expósitos de Barcelona.

Palabras clave: nodriza, inclusa, expósito, pobreza, Casa de Maternidad y Expósitos de Barcelona.

\begin{abstract}
This article analyzes wet nurses characteristics, their social consideration, their influence on mortality rates among the foundling and their role inside this charitable institutions and Casa de Maternidad y Expósitos de Barcelona.

Key words: wet nurse, foundling-hospital, foundling, poverty, Casa de Maternidad y Expósitos de Barcelona.
\end{abstract}




\section{Introducción}

En este artículo se estudia un tipo de trabajo que nada más podían hacerlo mujeres, el de nodriza de una inclusa, y se centra en la Casa de Maternidad y Expósitos de Barcelona (a partir de ahora, CMEB) en los años 1853-1903.

El trabajo de las amas internas y externas de las inclusas era la base sobre la que se sustentaba el funcionamiento de estos establecimientos benéficos. Estas mujeres eran imprescindibles porque, dados los malos resultados de la lactancia artificial en aquella época, sin su leche los expósitos en su totalidad hubieran fallecido. Además, ellas permitían la supervivencia de estas instituciones, pues en un porcentaje muy alto las nodrizas externas se quedaban con los niños que habían lactado, incluso cuando no cobraban por ellos por haber superado éstos la edad de los cinco o los siete años, según lo reglamentado por los distintos establecimientos benéficos. Sin esta actuación de estas mujeres, la capacidad de las inclusas y sus recursos económicos serían totalmente insuficientes para conceder a los expósitos una mínima posibilidad de sobrevivir. Sin embargo, el trabajo de las amas de las inclusas no estaba valorado y ellas mismas eran criticadas y menospreciadas por los órganos directores de estas instituciones, por la prensa, por los médicos, por los tratadistas, etc. En este artículo veremos cuáles eran los motivos, además de las características y la situación de estas trabajadoras.

Respecto a las nodrizas de la CMEB, dos libros publicados estos últimos años sobre la CMEB las analizan brevemente. Josep Montiel (Aguilar, Montiel, 2004: 41-3) señala su pobreza y las irregularidades que cometían, y Montserrat Alay (Alay, 2003: 111-5) incide sobre la escasez crónica de amas en la Institución y su influencia sobre la mortalidad de los expósitos. Una tesis doctoral leída en el año 2005 estudia más ampliamente este grupo de mujeres (Rodríguez, 2005: 458-490) y la postura de la junta de gobierno de la CMEB, que desviaba hacia ellas las responsabilidades del porcentaje de mortalidad tan elevado de los asilados. Este artículo pretende completar la visión sobre este tipo de trabajadoras que, además, formaban un grupo muy numeroso. 


\section{Las Nodrizas}

En el siglo XVIII, la lactancia mercenaria, mayoritariamente rural, estaba muy generalizada en algunos países europeos como Inglaterra, Alemania y, sobre todo, Francia. Pero en este mismo siglo, algunos médicos y publicistas empezaron a desaconsejarla porque opinaban que suponía un peligro para la vida de los niños, pues los ponía en contacto con mujeres mal alimentadas, y a veces enfermas, que vivían en casas sin las debidas condiciones higiénicas. Ya en el siglo XIX, los defensores de la lactancia materna, médicos sobre todo, la presentaban como una obligación moral y natural de las progenitoras y criticaban tanto a las mujeres que ejercían como nodrizas, pues comerciaban con su leche, es decir, con su cuerpo, como a las que recurrían a ellas. Este discurso fue calando en la sociedad de los países anteriormente citados e hizo que las madres de clase alta, las primeras, fueran abandonando lentamente el nodrizaje asalariado, sobre todo el realizado en la propia casa del ama de leche, que solía ser rural. Muchas de las mujeres que siguieron utilizando nodrizas las obligaron a que vivieran en el domicilio del niño para así controlarlas (Mause, 1982: 446-9; Gelis, 1978: 158-171). Otras madres, que no pertenecían a los grupos acomodados, empezaron a dejar a sus hijos en las casas de las amas de leche mientras iban a trabajar. Por todo ello, y a pesar de los detractores de la lactancia mercenaria, en algunos países como Francia, todavía a principios del siglo XX, el descenso en el porcentaje de niños puestos al cuidado de una nodriza no era demasiado importante. De los nacidos cada año, un tercio eran amamantados por amas, y muchos morían muy pronto, como ocurría también en el siglo anterior. Por ello, en 1874 se había aprobado la Ley Roussel que establecía la supervisión de los poderes públicos sobre las nodrizas (Hauser, 1902: 455-6; Rollet, 1982: 576-7).

También en España, en el siglo XVIII, un grupo de médicos abogó por la lactancia materna. En el siglo XIX sus partidarios aumentaron y, además, arreciaron las críticas a las amas de leche. Si a todo ello le unimos que a finales de siglo mejoró la lactancia artificial, se elevó el nivel de vida general y más mujeres se incorporaron al 
trabajo asalariado en las ciudades, la consecuencia fue que el mercado de amas se estrechó, aunque ligeramente (Guerra, 1887: 5-29. Pérez, 1972: 153-7. Vega, 1992: 578. Fraile, 2000: 29-30; Fuentes, 1997: 84-7). No obstante, las clases acomodadas seguían contratando nodrizas, que eran percibidas como un signo de distinción social, y no las ocultaban. En las calles más elegantes de Madrid y Barcelona era frecuente ver a las amas de leche con sus trajes de paseo. Las más apreciadas eran las del norte de España, y entre ellas, las del Valle del Pas. Estas mujeres pertenecían a las familias más pobres de su comunidad, y era precisamente su situación económica la que las empujaba a emigrar y a ejercer este tipo de trabajo (Soler, 2005: 223).

\section{Las Nodrizas de las Inclusas}

En las inclusas españolas, las nodrizas externas eran el eje sobre el que descansaba su funcionamiento, pues los niños se les entregaban al poco tiempo de haberse producido su ingreso, con ellas estaban hasta los cinco o los siete años e, incluso, muchas se los quedaban después de esta edad aunque ya no cobraran nada por su cuidado. Este papel de las nodrizas apenas sufrió cambios a lo largo de la época que estudiamos, 1853-1903.

Las amas podían ser internas o externas. Las primeras residían en la misma inclusa y su misión era lactar a los niños ingresados hasta que eran entregados a las amas externas. Éstas eran de lactancia o de destete. Las primeras alimentaban y cuidaban de los niños hasta los dieciocho o veinticuatro meses, según las inclusas, y eran las que tenían un salario más alto.

Las amas externas de las inclusas eran criticadas por algunos escritores de la época que les reprochaban que tuvieran su casa sucia y mal ventilada, que hicieran dormir a los niños en la misma cama que ellas, que no les proporcionaran instrucción ni principios morales y que no dedicaran apenas tiempo a su cuidado. En la mayoría de los casos, no se trataba de que dieran un trato especialmente malo a los expósitos, sino que 
era el mismo que recibiría un hijo suyo, que tendría que vivir en las mismas condiciones y al que no podría dedicar mucho tiempo, pues estas mujeres tenían que ocuparse de las faenas del campo, de la huerta y del ganado (Sarasúa, 1994: 147 y 187-193; González, 2001: 339-342). En el caso de las amas internas, las críticas procedían del hecho de que, generalmente, eran madres solteras y que, incluso, algunas dejaban a su propio hijo en la inclusa tras abandonar su trabajo.

Las nodrizas más sanas y acomodadas eran contratadas por particulares que pagaban más, por lo que las peores del mercado, las más pobres, débiles e, incluso, enfermas eran las que trabajaban para las inclusas españolas. Por esta razón estas mujeres tenían mala consideración social y los órganos directores de las casas de expósitos y algunos médicos se quejaban del descuido con el que trataban a los niños a su cargo (Novella, 1882: 130; Vidal, 1999: 84-5; Valverde, 1994: 160-1 y 173). Concepción Arenal describía a las amas insistiendo en la falta de condiciones que tenían para amamantar y en su influencia sobre los expósitos.

"Salvas algunas excepciones, las nodrizas de la inclusa o son de constitución poco robusta, o padecen algún achaque que puede transmitirse con la leche, o debiendo al vicio ser madres, y al crimen no tener hijos, envenenan a la vez el cuero y el alma de la mísera criatura que amamantan... La Beneficencia retribuye a las nodrizas con un salario que suele ser la mitad del que ganan en las casas particulares, el resultado no es difícil de prever"”.

En un intento de mejorar la conducta de las amas algunos reglamentos de estos centros benéficos establecieron premios y castigos para ellas ${ }^{2}$, pero esta medida no era efectiva porque, en la realidad, no se las sometía a una inspección directa y sólo se les reclamaban los expósitos a su cargo si existían malos tratos graves comprobados. Las

\footnotetext{
${ }^{1}$ Arenal, A. (1861): La beneficencia, la filantropía y la caridad. Madrid.

${ }^{2}$ Reglamento de la Casa de Maternidad y Espósitos de la Provincia de Lugo. Lugo, 1863, art. 106.
} 
inclusas tenían este bajo nivel de exigencia porque se encontraban siempre con escasez de nodrizas dado el salario tan corto que pagaban, que permanecía invariable durante años y que, en algunas ocasiones, era librado con mucha irregularidad (Casassas, 1993: 97. Gutiérrez, 1990: 188. Krause, 1990: 273. Álvarez, 1978: 121-3). Este sueldo era sensiblemente inferior al que recibían las mujeres que trabajaban en el sector textil y en el servicio doméstico (Sarasúa: 224-5). Además, las nodrizas tenían que sacar de su salario el dinero necesario para pagar el médico y las medicinas si el niño a su cargo enfermaba. Aunque en 1864 se estableció por Real Orden que los facultativos atenderían gratis a los expósitos, no siempre ocurría así ${ }^{3}$, por lo que éste era un motivo más para que la mayoría de las amas prefirieran lactar a un niño particular y para que de forma crónica las inclusas tuvieran escasez de nodrizas. Para intentar solucionar este problema las Juntas Provinciales de Beneficencia, y a partir de 1868 las Diputaciones Provinciales, insertaban notas en los Boletines Oficiales de la Provincia instando a los alcaldes, curas y facultativos a que colaboraran en su suministro. Cuando ya no se encontraban amas en la propia provincia, se buscaban en las limítrofes e, incluso, en otras regiones ${ }^{4}$. La escasez de nodrizas hubiera podido resolverse aumentando regularmente los salarios, tal como hizo la inclusa de Guipúzcoa cuando se le fue presentando el problema a lo largo del siglo XIX, pero la mayoría de estos establecimientos benéficos no adoptaron esta medida.

En las inclusas españolas toda mujer que quisiera lactar un asilado tenía que presentar un certificado médico de la calidad, abundancia y antigüedad de su leche. Además, el párroco y el alcalde tenían que acreditar la buena conducta de la aspirante. Sin embargo, había excepciones. En la de Barcelona, a las externas, además de los documentos citados se les reclamaba el certificado de defunción de su hijo para evitar sustituciones de niños o algún tipo de engaño de índole parecido. Sin embargo, en la de Navarra sólo se pedía el certificado parroquial. Respecto al estado civil, en la mayoría de las inclusas era condición imprescindible para ser ama el estar casada. Pero también

\footnotetext{
${ }^{3}$ Archivo Histórico de la Diputación de Barcelona (a partir de ahora, AHDB), leg. j-3.023.

${ }^{4}$ AHDB, leg. j-2.027 y j-3.024.
} 
había excepciones. En la de Barcelona sólo se exigía a las externas, y en la de Pontevedra a ninguna, creemos que debido al altísimo índice de ilegitimidad existente en Galicia (Uribe, 1996: 232; Rodríguez, 2003: 187. Rodríguez, 2005: 462-3; Barreiro, 1984: 165-7; González, 1905: 417).

\section{Las Nodrizas externas de la CMEB}

La Casa de Maternidad y Expósitos de Barcelona nació el 14 de noviembre de 1853 bajo la supervisión de la Junta Provincial de Beneficencia en unos locales de la calle Ramalleres. Antes de esa fecha los expósitos estaban acogidos en el Hospital de la Santa Cruz, que tenía un departamento destinado a ellos. En 1885 los niños de destete y los mayores de cinco años fueron trasladados a los nuevos locales de la Institución ubicados en la finca de Can Caballé, en el municipio de Les Corts, vecino al de Barcelona. Por fin, en 1892, también allí fueron trasladados los de lactancia ${ }^{5}$.

En la CMEB, lo mismo que en el resto de las inclusas españolas (Valverde, 1994: 182-3; Junceda, 1984: 88), los niños eran entregados mayoritariamente a nodrizas externas rurales, pues se consideraba que era beneficioso para ellos integrarse en una familia y vivir en el campo. Los niños expuestos o abandonados en una comarca determinada nunca eran dados a un ama allí residente ni a la mujer que les hubiera conducido a la Institución, pues se buscaba evitar fraudes y preservar el secreto de la identidad de los acogidos ${ }^{6}$.

A las nodrizas la CMEB les exigía ser casadas o viudas y presentar dos certificados, uno médico y otro de su alcalde. Esta documentación era imprescindible y

\footnotetext{
${ }^{5}$ AHDB, leg. j-3.024 y 1.350 al 1.352. Sesiones de la Junta de Gobierno de la CMEB, 27-6-1885, 8-11-1889 y 3-12-1889. AHDB, leg. j-2.028.

${ }^{6}$ Reglamento de la Casa Hospicio y Niños Expósitos de la Provincia de Burgos. Burgos, 1863, art. 92. "Real Casa de Beneficencia y Maternidad de La Habana". En Revista de Beneficencia, $\mathrm{n}^{\circ}$ 20, 8-1-1888, pp. 3-4. Reglamento para el régimen y gobierno del Gran Hospital de Santiago y de la Casa de Expósitos anexa al mismo. La Coruña, 1881, art. 241.
} 
sin ella no se entregaba expósito alguno, lo mismo que si el ama no tenía domicilio fijo. Aunque el Reglamento de 1890 de la CMEB establecía la revisión de las nodrizas por parte de un facultativo de la Institución, esto no se llevó a cabo. Las amas de destete no tenían que presentar tanta documentación, sólo el certificado de buena conducta firmado por el alcalde o el párroco, en el que, además, tenían que constar sus medios de subsistencia ${ }^{7}$.

El número de nodrizas de la CMEB era muy alto, como podemos ver en el cuadro número 1. En los primeros años de vida de la Institución eran más numerosas las de lactancia. Sin embargo, en la década de los ochenta, cuando ya era clara la disminución de la mortalidad de los expósitos, las más abundantes eran las de destete.

Cuadro 1. Número de amas externas de la CMEB, 1860-1888 ${ }^{8}$

\section{Número de amas}

\begin{tabular}{lccc} 
Año & lactancia & destete & total \\
\hline $\mathbf{1 8 6 0}$ & 851 & 730 & 1.581 \\
$\mathbf{1 8 6 5}$ & 741 & 836 & 1.577 \\
$\mathbf{1 8 8 8}$ & 669 & 760 & 1.429 \\
\hline
\end{tabular}

La CMEB únicamente pagaba, y por trimestres vencidos, a las amas externas que presentaran la hoja de lactancia en la que constara el certificado del párroco conforme el expósito se hallaba bien de salud y llevaba al cuello el plomo o medalla con su número de inscripción en la Institución. Los salarios, como podemos observar en el cuadro que sigue, eran muy bajos, pero significaban la entrada de dinero en metálico en familias muy pobres, aportado por la mujer y no por el cabeza de familia.

\footnotetext{
${ }^{7}$ Reglamento especial para las Casas unidas de Maternidad y Expósitos de la ciudad de Barcelona. Barcelona, 1853, art. 25. Reglamento de la Casa Provincial de Maternidad y Expósitos de Barcelona. Barcelona, 1890, art. 87. AHDB, leg. j-3.023, j-3.027 y j-3.028.

${ }^{8}$ AHDB, leg. j-3.022, j-3.024 y j-3.027.
} 
Cuadro 2. Salarios mensuales de las amas de la CMEB, 1853-1903 ${ }^{9}$

\section{Nodrizas externas}

$\begin{array}{llr}\text { Años } & \text { lactancia } & \text { destete } \\ \mathbf{1 8 5 3 - 6 4} & 50 \text { reales } & 25 \text { reales } \\ \mathbf{1 8 6 7} & 60 \text { escudos } & 30 \text { escudos } \\ \mathbf{1 8 7 9} & 15 \text { pesetas } & \text { sin datos } \\ \mathbf{1 8 8 7 - 1 9 0 3} & 20 \text { pesetas } & 6,25 \text { pesetas }\end{array}$

Dado que las familias hacían una competencia muy grande a las inclusas a la hora de la contratación, ya desde el comienzo de la vida de la CMEB, la mayoría de las amas externas residían fuera de Barcelona capital. Pero incluso estas mujeres, en cuanto les salía una lactancia particular, devolvían a los expósitos ${ }^{10}$. Otras muchas preferían otro tipo de trabajo. En Barcelona, a principios del siglo XX, hasta las sombrereras, lavanderas, modistas, planchadoras, etc. cobraban más. Solamente las profesiones peor pagadas, que eran las de niñera, alpargatera y guantera, podían equipararse a la de nodriza $^{11}$. Pero no era únicamente el bajo salario lo que disuadía a muchas mujeres de ser amas de la CMEB. Pesaba mucho el miedo a ser contagiadas de alguna enfermedad venérea por el expósito al que lactasen. La más temida era la sífilis que, como tardaba en mostrar síntomas, si no era detectada por los servicios médicos de la Institución, el expósito afectado salía a lactancia externa y suponía un peligro para su ama. Las inclusas no tenían obligación legal de asistir a las contagiadas, pero sabemos que la CMEB, al menos en tres casos, no se desentendió de ellas. En el primero, en 1885, ofreció el tratamiento gratuito de la enfermedad en la propia Institución o una determinada cantidad de dinero. En el segundo caso, en 1905, la ayuda fue miserable.

\footnotetext{
${ }^{9}$ AHDB, leg. j-2.206 y j-3027 al j-3.234. Rull,J. y Cabot, J. Estudio demográfico-médico del quinquenio de 1883 a 1887 de la Casa Provincial de Maternidad y Expósitos de Barcelona. Barcelona, 1889, p. 27.

${ }^{10}$ AHDB, leg. j-2.206, j-3.030 y j-3.027. "La Casa de Maternidad". En El Liberal, no 952, 22-111903, p. 13.

11 Estadísticas sociales. Monografía estadística de la clase obrera. Barcelona, 1921, Ayuntamiento de Barcelona, pp. 100-1.
} 
Se trataba de una nodriza que pedía únicamente sesenta pesetas para enviar a sus tres hijos a casa de sus padres, pues tenía miedo a contagiarles. La CMEB accedió a comprarle los billetes, pero siempre que no pasaran de la cantidad citada. En el tercer caso, el ama, incluso, había transmitido la enfermedad a su esposo. Su única petición, que le fue concedida, consistió en que su marido recibiera tratamiento en el Hospital de la Santa Cruz de Barcelona ${ }^{12}$.

\subsection{Las características de las nodrizas}

En el siglo XIX, la mayoría de las amas externas de las inclusas españolas residían en zonas rurales, aunque había excepciones, como en Madrid o Sevilla. En esta última capital tenían su domicilio en los barrios más pobres. Respecto a las nodrizas de la CMEB, las pocas que eran vecinas de Barcelona capital vivían en una gran proporción en la Barceloneta, como podemos observar en el cuadro número 3, un distrito compuesto por casas pequeñas e insanas en una zona industrial donde no había cloacas y donde imperaba la pobreza. Las enfermedades como la viruela, la enterocolitis y el cólera eran muy frecuentes, y la mortalidad infantil era muy alta, lo mismo que la de población en general, lo que colocaba a este barrio entre los tres con más fallecimientos de Barcelona. La necesidad de un ingreso, por mínimo que fuera, empujaba a muchas mujeres que habían perdido a su hijo a lactar a un expósito (Tatjer, 1995: 127-9 y 132-4; Colomer, 1883: s/p).

\footnotetext{
${ }^{12}$ AHDB, leg. j-2.434.
} 
Cuadro 3. Residencia en Barcelona capital de las amas externas de la CMEB, 1854-1870 13

\section{La Barceloneta}

\section{Resto de distritos}

\begin{tabular}{lcccc} 
Año & número & porcentaje & número & porcentaje \\
\hline $\mathbf{1 8 5 4}$ & 4 & 16,7 & 20 & 83,3 \\
$\mathbf{1 8 6 4}$ & 24 & 40 & 36 & 60 \\
$\mathbf{1 8 6 7}$ & 17 & 35,4 & 31 & 64,6 \\
$\mathbf{1 8 7 0}$ & 24 & 34,3 & 46 & 65,7
\end{tabular}

Si consideramos solamente a las nodrizas de la provincia de Barcelona que la CMEB iba contratando a lo largo de cada año, las residentes en la Capital, y en los cincuenta años que analizamos, fueron siempre menos de la mitad, a pesar de que en 1900 vivía en ella el 50,6 \% de la población de toda la Provincia ${ }^{14}$.

Aunque a los pocos días de vida de la CMEB su Junta de Gobierno tomó la decisión de que sólo pudiesen ser amas externas las vecinas de la ciudad de Barcelona y de su Provincia ${ }^{15}$, la falta de aspirantes a este trabajo hizo que este acuerdo fuera papel mojado y que tuviera que contratar a nodrizas de las provincias limítrofes e, incluso, de otras regiones. Solamente los años siguientes a las subidas de sueldo la Institución tenía la oferta de amas suficiente como para poder elegir mínimamente (Rull, Cabot, 1889: 26-27). Sin embargo, conforme pasaba el tiempo y los salarios permanecían iguales, la oferta se iba haciendo menor y la CMEB tenía que contratar a las pocas mujeres que se presentaban para lactar expósitos. El problema se agravaba durante los veranos y las épocas de epidemias, pues eran pocas las que venían a Barcelona a recoger expósitos ${ }^{16}$.

En los primeros años de la época que estudiamos, 1853-1903, y dejando aparte Barcelona, Girona era la provincia de Cataluña que proporcionaba más nodrizas. Sin embargo, a partir de 1889 , Lleida y Tarragona tomaron el relevo ${ }^{17}$. Todas estas mujeres

\footnotetext{
${ }^{13}$ AHDB, leg. j-3.014, j-3.017, j-3.018 y j-3.024.

${ }^{14}$ AHDB, leg. j-3.220 y j-3.231 al j-3.234.

${ }^{15}$ Sesión de la Junta de Gobierno de la CMEB, 27-11-1853. AHDB, leg. j-2.027.

${ }^{16}$ AHDB, leg. j-3.023.

${ }^{17}$ AHDB, leg. j-3.220 y j-3.231 al j-3.234.
} 
vivían en el campo o en pueblos muy pequeños. Los motivos de la preferencia de las amas de estas tres últimas provincias citadas por lactar niños de la CMEB eran económicos. En 1860, el Hospicio de Girona pagaba mensualmente 20 reales y, sin embargo, la CMEB cincuenta. Cuando el Hospicio elevó los sueldos, las nodrizas dejaron de coger expósitos barceloneses (Puy, 2002: 130-1). En 1900, el 53,6\% de la población catalana vivía en la provincia de Barcelona (Recolons, 1974: 49, 62 y 171), un porcentaje parecido al de las amas de la CMEB que en 1903 residía en la misma provincia, como podemos ver en el cuadro número 4.

\begin{tabular}{|c|c|c|c|c|}
\hline \multirow[b]{2}{*}{$\underline{\text { Año }}$} & \multicolumn{2}{|c|}{ Provincia de Barcelona } & \multicolumn{2}{|c|}{ resto de las provincias catalanas } \\
\hline & número & porcentaje & número & porcentaje \\
\hline 1854 & 5 & 98,7 & 1 & 1,3 \\
\hline 1864 & 347 & 68,9 & 157 & 31,1 \\
\hline 1874 & 231 & 71,7 & 91 & 28,3 \\
\hline 1884 & 310 & 50 & 310 & 50 \\
\hline 1894 & 255 & 46,3 & 296 & 53,7 \\
\hline 1903 & 272 & 53,4 & 237 & 46,6 \\
\hline
\end{tabular}

Un tanto por ciento muy pequeño, pero que fue aumentando a lo largo de los años que analizamos, lo representan las nodrizas residentes en Aragón y en la Comunidad Valenciana. Si en 1854 no había ninguna de estas dos zonas, en 1903 se contabilizaban 34 de Aragón y 114 de la Comunidad Valenciana, que representaban el $5,2 \%$ y el $17,4 \%$, respectivamente, del total de las amas contratadas por la CMEB ese año ${ }^{19}$. En su mayoría eran de las provincias limítrofes con Cataluña, es decir, de Huesca y de Castellón, donde las mujeres tenían menos salidas profesiones y donde había más

\footnotetext{
${ }^{18}$ AHDB, leg. j-3.220 y j-3.231 al j-3.234.

${ }^{19}$ AHDB, leg. j-3.220 y j-3.231 al j- 3.234 .
} 
pobreza, pero donde el desarrollo de las comunicaciones hacía que Barcelona no quedara tan lejos. En la Inclusa de Navarra ocurría algo similar y por los mismos motivos, por lo que si en 1890 la práctica totalidad de las nodrizas residían en la Provincia, a inicios del siglo XX ya había amas de las provincias próximas, es decir, de Logroño, Soria y Zaragoza (Uribe, 1996: 217-228). En la Inclusa de Pontevedra el porcentaje de nodrizas residentes en la provincia de Lugo oscilaba, según los años, entre el 21 y el 57\% del total de amas (Rodríguez, 2003: 192). Sin embargo, en otras inclusas de provincias lo bastante pobres como para que resultara atractiva la lactancia de un expósito, todas las amas vivían en ella, como sucedía en la Casa Cuna de Sevilla.

Había una serie de municipios que se especializaban en el nodrizaje. Tenían en común su situación en zonas muy pobres y sin industria. En algunos casos, estaban localizados en territorios montañosos en los que era importante el sector ganadero, que permitía a las mujeres disponer de más tiempo para cuidar un expósito. En la CMEB la mayoría de estos municipios contaban con una conductora de expósitos, es decir, una mujer que recogía niños de la inclusa y los iba entregando a las nodrizas en su propio pueblo, aldea o masía. Cuántas más amas reclutase, cuantos más niños transportase y más hojas de lactancia presentase en la Inclusa para el cobro, más comisiones ganaba. Por esta razón, las conductoras se esforzaban en animar a las madres de su zona a lactar a un inclusero. En Barcelona, de los siete municipios de la Provincia que proporcionaron más nodrizas a la $\mathrm{CMEB}$, cuatro eran colindantes con la Ciudad, con la que se unieron en 1897, por lo que la proximidad fue un factor importante. Los tres restantes estaban situados en el interior de la Provincia ${ }^{20}$. Los municipios de la costa de Barcelona, así como los más industrializados como Terrassa o Sabadell, apenas proporcionaron amas a la CMEB. Su nivel de vida, superior al de los pueblos del interior, y las mayores oportunidades de trabajo para las mujeres hacían que las residentes no aceptaran nodrizajes.

\footnotetext{
${ }^{20}$ Los colindantes eran Gracia, Sant Martí de Provensals, Sans y Sant Andreu. Los del interior, Torrelles de Foix, Igualada y Manresa. AHDB, leg. j-3.230 al j-3.235.
} 
En la provincia de Girona, los seis municipios donde residían la mayor parte de las amas de esta demarcación que lactaban asilados de la CMEB estaban situados en el interior y no en la costa. En todos ellos la agricultura y, sobre todo, la ganadería ocupaba a la mayor parte de la población, frente a otros municipios, como el de La Bisbal, donde residían menos nodrizas y donde el porcentaje de la población dedicado al sector primario era menor ${ }^{21}$.

La especialización de zonas en la crianza de expósitos no sucedía únicamente en la CMEB. En la Inclusa de Pontevedra las nodrizas de la provincia de Lugo residían exclusivamente en los municipios de Pantón y Cerdedo, lugares donde la industria y el comercio eran prácticamente inexistente y donde la agricultura era poco productiva (Rodríguez, 2003: 193-4).

\subsection{La conducta de las nodrizas}

Las nodrizas externas de la CMEB eran controladas por sus párrocos, sin cuyo certificado conforme el expósito a su cargo se encontraba bien alimentado y con salud, no cobraban. Además, en cada pueblo cabeza de partido judicial había una comisión de señoras que vigilaba la conducta de las amas y en caso de falta grave le retiraban el niño ${ }^{22}$.

Algunas nodrizas, según la opinión de las personas que se encargaban de controlarlas, eran lo peor de la sociedad, mujeres "criminales" y "avariciosas" que, en algunos casos, habían pasado un tiempo en la cárcel, y cuya pobreza extrema las llevaba a mendigar y a arrastrar con ellas a los expósitos ${ }^{23}$. Estas amas, a pesar de no tener una vivienda en condiciones, de no disponer de leche abundante y de, en muchas ocasiones, no tener salud, solicitaban lactar a un inclusero porque era la única manera de conseguir

\footnotetext{
${ }^{21}$ Censo de la población de España según el empadronamiento verificado el 31-12-1887. Madrid, 1892, vol. II, pp. 144-7, 478 y 479.

22 Reglamento especial para las Casas unidas de Maternidad y Expósitos de la ciudad de Barcelona. Barcelona, art. 25-29, 51 y 55. AHDB, leg. j-3.023, j-3.024, j-3.027 y j-3.038.

${ }^{23}$ AHDB. leg. j-2.434, j-3.011, j-3.017, j-3.020, j-3.023 y j-3.112.
} 
unos ingresos regulares. Por esta misma razón, si el niño que recibían estaba débil o enfermo, lo que implicaba muchos cuidados y gastos en medicinas, era probable que lo devolvieran. Debido a esto, era muy frecuente que este tipo de expósitos tuviera tres, cuatro e incluso cinco nodrizas ${ }^{24}$. La CMEB no desconocía estas conductas de sus amas, pero dada la escasez y la cortedad del salario que pagaba, tenía que transigir y aceptar prácticamente a toda mujer que se ofreciera como nodriza, e incluso readmitir a aquéllas que la habían engañado. Estas amas tan pobres cometían todo tipo de fraudes, como ocurría en las otras inclusas españolas (Morales, 2001: 75; Fernández, 1996: 418). Uno de los más frecuentes era el traspaso de expósitos y le seguía la ocultación por parte de la nodriza del hecho de que se había quedado sin leche, o la tenía escasa y vieja, o se había quedado nuevamente embarazada, o bien había tenido con posterioridad un hijo propio al que debía lactar ${ }^{25}$. Estos casos los encontramos a lo largo de los cincuenta años que estudiamos y estaban favorecidos por el hecho de que la CMEB permitió, en 1859, que las amas pudieran cobrar su salario a través de personas autorizadas por ellas. Esta medida tenía como objetivo evitar el viaje hasta la Institución, a menudo largo y fatigoso, que tenían que hacer en las dos épocas de pago, julio y diciembre, las nodrizas y los lactantes. Sin embargo, esta disposición llevó consigo que no hubiera la posibilidad de que los médicos de la CMEB examinaran a estas mujeres y, desde luego, los de los pueblos tampoco lo hacían ${ }^{26}$. Las amas podían así ocultar el estado de su leche y si el párroco o las comisiones de señoras no denunciaban la situación, la CMEB no llegaba a enterarse.

A los seis meses de funcionamiento de la Institución, ésta ya pudo ver que algunos niños eran devueltos por sus nodrizas en muy mal estado. La CMEB hablaba de malos tratos, de la devolución de niños agonizantes o bien en un estado lastimoso:

\footnotetext{
${ }_{25}^{24}$ AHDB, leg. j-2.424, j-2.434 y j-3.112.

${ }^{25}$ AHDB, leg. j-2.028, j-3.017, j-3.020, j-3.027 al j-3.029, y j-3.038.

${ }^{26}$ AHDB, leg. j-3.023.
} 
“...era un esqueleto que ni cuasi forma humana tenía tanta era la miseria, el mal trato y hambre que había sufrido",27.

“...el estado cadavérico en que ha devuelto al referido expósito hace sospechar un comportamiento criminal" ${ }^{, 28}$.

“...está la nodriza amamantando a un expósito con tan malos resultados que está cuasi exánime, por manera que ha llamado la atención así de las vecinas..., 29 .

En mayoría de los casos el problema radicaba en que los expósitos habían recibido una escasa o mala alimentación. Las amas, mujeres extremadamente pobres, habían retrasado todo lo posible la devolución del niño para no quedarse sin el salario que recibían a cambio, por lo que, si se habían quedado sin leche, utilizaban la de cabra, o bien destetaban antes de tiempo al lactante. Otras se quedaban embarazadas y luego amamantaban al mismo tiempo a su hijo biológico y al expósito. Estas nodrizas no pretendían hacer un gran negocio, cosa imposible dado el sueldo tan escaso que cobraban sino, sencillamente, sacar el máximo dinero posible de su leche dada la miseria en la que vivían ${ }^{30}$. Por el mismo motivo, algunas amas traspasaban los expósitos a mujeres que por su conducta o por ser solteras nunca serían contratadas por la CMEB. En otras ocasiones el traspaso venía motivado porque a la nodriza le había surgido una lactancia particular o bien porque se había quedado embarazada. Entonces subcontrataba a otra mujer o le vendía la hoja de lactancia y el derecho al cobro que llevaba consigo. Frecuentemente esta operación implicaba que ejercía de ama una con peores condiciones que la titular, que tenía menos leche o que amamantaba a la vez a su hijo biológico, o que estaba en la miseria más absoluta ${ }^{31}$. Veamos una actuación de este tipo. En 1861 una nodriza cobraba 50 reales de la CMEB, pero se quedó embarazada,

\footnotetext{
${ }^{27}$ Escrito de la CMEB al Gobernador Civil, 16-10-1855. AHDB, leg. j-3.023.

${ }^{28}$ Sesión de la Junta de Gobierno de la CMEB, 8-10-1860. AHDB, leg. j-2.027.

${ }^{29}$ Escrito del párroco de Sabadell a la CMEB, 1865. AHDB, leg. j-3.029.

${ }^{30}$ AHDB, leg. j-3.020 y j-3.023.

${ }^{31}$ AHDB, leg. j-2.431, j-3.020, j-3.027, j-3.029 y j-3.038.
} 
por lo que subcontrató a otra ama para que lactase por 40 reales al expósito a su cargo, a la que se lo reclamó tras ocho meses. Pero entonces no lo alimentó con su propia leche, pues la reservaba para su hijo, sino con leche de burra ${ }^{32}$. Se trataba de estratagemas de supervivencia de mujeres muy pobres, aunque la CMEB sólo veía en estas actuaciones codicia:

“...hechos de que se ocupará luego la propia Dirección...vienen a justificar la necesidad imperiosa que hay de tomar medidas severas para que estos pobres expósitos no sirvan de instrumento a la codicia y especulación de algunas personas desalmadas, que afortunadamente son pocas..."33.

Frente a estas actuaciones, la CMEB no tomó medida especial alguna. Si el traspaso había sido temporal, no se exigía la devolución del expósito porque los mismos párrocos y alcaldes comunicaban a la Institución la dificultad para encontrar otra nodriza. Si el traspaso era ya definitivo, se reclamaba el niño en cuestión y no se pagaba salario alguno al ama.

No podemos asegurar que los casos que hemos visto eran aislados o bien se trataba de la punta del iceberg. Los encargados de supervisar la conducta de las nodrizas eran los párrocos, las comisiones de señoras y los alcaldes ${ }^{34}$. Su dedicación era variable y, además, las comisiones de señoras no llegaban a supervisar a las amas rurales, que eran la mayoría. La propia Institución no realizaba inspecciones directas. Sólo tenemos constancia de alguna de ellas a las comarcas donde residían más nodrizas y se saldaron con la reclamación de varios expósitos que recibían mala alimentación ${ }^{35}$. Otros, que ya

\footnotetext{
${ }^{32}$ AHDB, leg. j-3.020 y j-3.028.

${ }^{33}$ Escrito de la CMEB al Gobernador Civil, 16-10-1855. AHDB, leg. j-3.023.

${ }^{34}$ Bodas de Oro de la Casa de Maternidad y Expósitos de Barcelona. Barcelona, 1903, p. 10. AHDB, leg. j-3.020.

${ }^{35}$ AHDB C.C./VII 610 y j-3.020. Sesiones de la Junta de Gobierno de la CMEB, 29 de mayo, 20 de junio y 15 de septiembre de 1891, y 14-10-1896. AHDB, leg. j-2.028.
} 
no eran lactantes, compartían la pobreza de sus amas e incluso eran llevados a mendigar, solos o con ellas mismas ${ }^{36}$.

Algunas de las enfermedades de las que morían los expósitos, como el escorbuto o la tuberculosis, nos indican la miseria en la que vivían las nodrizas ${ }^{37}$. Esta misma circunstancia provocaba que cuando el niño enfermaba no se llamara al médico y se le tratara con recetas de medicina casera o supersticiones. Una de estas creencias populares era que la diarrea limpiaba los intestinos, por lo que las amas no le daban importancia a esta dolencia. Cuando consideraban que duraba ya demasiado intentaban cortarla con remedios caseros que no solían tener éxito, por lo que cuando intervenía el médico ya era demasiado tarde (Aguirre, 1885: 144). Algunas veces, como entregar el niño suponía perder el mísero salario, lo hacían cuando la enfermedad estaba avanzadísima, y por eso, y a lo largo de los cincuenta años que estudiamos, encontramos expósitos que mueren de tuberculosis en la Institución a los pocos días de haber reingresado ${ }^{38}$.

Estas conductas negativas de las nodrizas eran conocidas públicamente y se reflejaban en las revistas ${ }^{39} \mathrm{y}$, en el caso de la $\mathrm{CMEB}$, incluso en la prensa diaria:

"Según los datos que nos han sido suministrados...la mayoría de las nodrizas en cuyas manos están estos desgraciados expósitos, unas no tienen la cantidad de leche necesaria para que los niños se puedan nutrir, debido a que antes de lactar al expósito que les ha sido entregado, ya llevan tres o más años de amamantar a los suyos propios, o bien a otros de casas particulares; y en cuanto a otras, menos escrupulosas todavía y movidas por su afán de lucrarse a costa del prójimo, ....son unas amas completamente secas" ${ }^{\natural 0}$.

\footnotetext{
${ }^{36}$ AHDB, leg. 763, j-3.017 y j-3.023.

${ }^{37}$ AHDB, leg. j-2.298.

${ }^{38}$ AHDB, leg. j-3.083, 3.093 y j-3.112.

39 "Lactancia mercenaria", en Revista de Beneficencia, no 18, 22-12-1887, p. 3.

${ }^{40}$ Artículo sin título. El Diluvio, Barcelona, no 304, 30-10-1896, pp. 5-6.
} 
Cuando un menor era devuelto por su nodriza en mal estado, los facultativos de la CMEB lo reconocían y, en algunos casos, aconsejaban que se pidiera información al alcalde y al párroco del lugar de residencia del ama sobre si ésta había alimentado bien al expósito y si lo había llevado al médico cuando se había puesto enfermo. Si se apreciaba negligencia por parte de la nodriza pero el niño no fallecía, la CMEB se limitaba a no pagarle su salario. Igual sanción se imponía a las que habían lactado escasamente a los expósitos a su cargo y a las que los habían destetado demasiado pronto. La Junta de Gobierno de la Institución creía que los castigos a las amas eran muy leves pero recurría pocas veces a la Justicia debido a la escasez de nodrizas ${ }^{41}$. También creemos que en una época en la que la mortalidad infantil era tan alta, la muerte de unos incluseros no se juzgaba tan grave.

El trato cuidadoso de las nodrizas de las inclusas y sus familias hacia los expósitos también existió, pero tenemos menos información sobre ello porque era lo normal. Las malas conductas eran, precisamente, las que generaban más documentación.

El primer testimonio de afecto y lealtad de un ama hacia el expósito a su cargo era su devolución, por voluntad propia, cuando la mujer se daba cuenta de que estaba embarazada, su leche era de mala calidad, escasa o se había quedado sin ella. Estos casos fueron continuos a lo largo de los cincuenta años de vida de la CMEB que estudiamos, así como los de nodrizas que comunicaban a la Institución que se encontraban enfermas y que la pobreza de la familia no les permitía cuidar adecuadamente a los incluseros a su cargo ${ }^{42}$.

En la CMEB, cuando los expósitos eran legitimados, naturalizados ${ }^{43}$, o reclamados por la Institución, las amas tenían que devolverlos, aunque llevaran muchos años conviviendo. En bastantes casos estas nodrizas habían hecho de verdaderas madres

\footnotetext{
${ }^{41}$ AHDB, leg. j-3.023.

${ }^{42}$ AHDB, leg. j-3.020, j-3.029 y j-3.038.

${ }^{43}$ Se producía la legitimación cuando mediante escritura pública unos padres casados reconocían a su hijo. La naturalización era el reconocimiento público de un hijo realizado por un hombre o una mujer, o por ambos, pero sin ser matrimonio.
} 
y se habían quedado los niños, a pesar de haber cumplido éstos los cinco años y de no percibir salario alguno por ellos. Algunas de estas mujeres, aún teniendo hijos propios y viviendo en la miseria, no querían deshacerse de su expósito ${ }^{44}$. En este sentido informaba un párroco en 1895, “...la familia es tan pobre que a duras penas puede mantener y educar a sus hijos propios; y no obstante de haberles requerido varias veces la devolución del expósito a esa Casa, no cuidan hacerlo" ${ }^{\text {,45. }}$

El porcentaje de amas a las que la CMEB confió niños, es decir, permitió que conservaran a su lado a incluseros que habían lactado, fue muy alto, como podemos ver en el cuadro que sigue.

Cuadro 5. Expósito mayores de cinco años, por generaciones, que se quedaron a vivir con sus amas, $1854-1903^{46}$

\section{Expósitos confiados a sus amas}

\begin{tabular}{lcccc} 
Año & ingresados & supervivientes & número & \% sobre supervivientes \\
\hline $\mathbf{1 8 5 4}$ & 709 & 152 & 45 & 29,6 \\
$\mathbf{1 8 5 9}$ & 747 & 209 & 116 & 55,5 \\
$\mathbf{1 8 6 4}$ & 729 & 198 & 119 & 60,1 \\
$\mathbf{1 8 6 9}$ & 704 & 184 & 115 & 62,5 \\
$\mathbf{1 8 7 4}$ & 653 & 114 & 62 & 54,4 \\
$\mathbf{1 8 7 9}$ & 677 & 190 & 86 & 45,3 \\
$\mathbf{1 8 8 4}$ & 649 & 250 & 136 & 54,4 \\
$\mathbf{1 8 8 9}$ & 590 & 278 & 158 & 56,8 \\
$\mathbf{1 8 9 4}$ & 671 & 306 & 159 & 52 \\
$\mathbf{1 8 9 9}$ & 623 & 284 & 161 & 56,7 \\
$\mathbf{1 9 0 3}$ & 600 & 290 & 164 & 56,5
\end{tabular}

\footnotetext{
${ }^{44}$ AHDB, leg. j-3.020.

${ }^{45}$ AHDB, leg. j-3.027.

${ }^{46}$ AHDB, leg. j-3.062 al j-3.112.
} 
Muchos de los niños a cargo de las nodrizas fueron tratados como hijos propios y recibieron cuidados en sus enfermedades, incluso en las de larga duración. En algún caso tuvo que intervenir el médico del pueblo para obligar al ama y a su esposo a que devolvieran a la CMEB el expósito a su cargo:

"En los dos años que dura la dolencia (de la expósita) los esposos...se han desvelado en cuidar a la paciente...robando horas a su descanso y sacrificándose en sus intereses, pero atendiendo a la duración de la enfermedad...y a la carencia de recursos de los encargados de la paciente, opino que para que no le falten los medios farmacológicos, higiénicos y dietéticos convenientes es necesaria su traslación a la Casa..." ${ }^{47}$.

Hubo nodrizas que cuando enviudaban y se volvían a casar, llevaban consigo al nuevo matrimonio el niño que cuidaban. Algunas, si decidían irse al extranjero a trabajar y no obtenían el permiso de la CMEB para llevarse al expósito que cuidaban, suspendían definitivamente el viaje. Si la Institución ponía condiciones para permitir la salida de España, las amas y sus esposos se apresuraban a cumplirlas ${ }^{48}$. Otras muchas amas a pesar de los problemas que los expósitos, ya adolescentes, les ocasionaban no se desprendieron de ellos ${ }^{49}$. Además, tenemos constancia de que algunas de estas mujeres y sus consortes les nombraron herederos en sus testamentos o les legaron parte de sus escasos bienes.

Cuando los expósitos eran reclamados por la CMEB, muchas nodrizas sufrían enormemente con la separación e intentaban anular la orden de devolución ofreciendo dejarles en herencia todo lo que poseían, que era muy poco pues se trataba de mujeres muy pobres ${ }^{50}$ :

\footnotetext{
${ }^{47}$ Escrito del médico de Manresa a la CMEB, 18-8-1901. AHDB, leg. j-2.394.

${ }^{48}$ AHDB, leg. j-2.028, j-3.028 y j-3.029.

${ }^{49}$ AHDB, leg. j-3.023.

${ }^{50}$ AHDB, leg. 763, j-3.020 y j-3.038.
} 


\begin{abstract}
"Ella hace oferta de dejarle una casa y huerto, y los bienes muebles...pero la oferta que ella hace es una cosa ficticia, pues todos sus bienes consisten en un pequeño casucho y poco más o menos media cuarta de terreno...sus muebles son tan insignificantes que no valen la pena de mencionar" ${ }^{\text {. }}$.
\end{abstract}

Otras amas se resistían a devolver el expósito a su cargo y por ello ponían todo tipo de trabas. La más frecuente era la de que no tenían dinero para el viaje a Barcelona o que se encontraban enfermos el niño o ella. También buscaban las pocas influencias que podían tener y hacían que el párroco o el alcalde de su pueblo escribieran a la CMEB hablando en su favor e, incluso, reunían firmas de sus vecinos que apoyaran su petición. En último caso, recurrían al cambio de domicilio para que la Institución no pudiera localizarlas ${ }^{52}$. Cuando las reclamaciones de expósitos se hacían efectivas, la CMEB era inflexible e impedía todo contacto posterior entre estos niños y las que habían sido sus nodrizas.

\title{
5. Las amas internas de la CMEB
}

En la CMEB, al igual que en las otras inclusas españolas ${ }^{53}$, el número de nodrizas internas no era muy elevado, puesto que los niños eran dados a las externas a los pocos días de ingresar. Aunque las entradas diarias de expósitos, a lo largo del período que consideramos, experimentaron un ligero descenso, la cantidad de amas internas aumentó. En la primera mitad de la época que analizamos, 1853-1903, fluctuó

\footnotetext{
${ }^{51}$ Oficio del alcalde de la nodriza M.P. a la Junta Provincial de Beneficencia, 13-8-1871. AHDB, leg. 763.

${ }_{53}^{52}$ AHDB, leg. j-2.395, j-2.401, j-3.017, j-3.020 y j-3.038.

53 "Expósitos y enfermos". En Revista de Beneficencia, no 4, 15-9-1887, p. 3. "Miscelánea". En Revista de Beneficencia, $\mathrm{n}^{\circ}$ 16, 8-12-1887, p. 5. Reglamento para el régimen interior administrativo y económico del establecimiento provincial de Espósitos de Málaga. Málaga, 1856, art. 21-31.
} 
entre 30 y 50, y en la segunda entre 50 y $60^{54}$. Esto se debió a que desde 1880, y de forma más generalizada a partir de 1892, los niños permanecían más tiempo en la Institución antes de ser entregado a las nodrizas externas, para así detectarles las enfermedades que pudieran portar, como la sífilis.

El trabajo de ama interna tenía algunas ventajas. Proporcionaba un techo y alimentación abundante y rica en proteínas ${ }^{55}$. Es verdad que durante los primeros 30 años que nosotros analizamos la CMEB estuvo ubicada en la calle Ramalleres, en un local que no reunía las debidas condiciones, pero no era peor que el que podía esperar una sirvienta que, en muchas ocasiones, tenía que compartir el cuarto más insalubre de la vivienda en la que trabajaba con las otras criadas. No obstante, la CMEB se encontró siempre con el problema de la escasez de nodrizas internas debido a que las lactancias particulares se pagaban mejor. Además influía la falta de libertad que había en la Institución. Aunque en los primeros días de su funcionamiento las amas internas, tras amenazar con abandonar el trabajo, consiguieron el permiso para salir unas horas el martes y el sábado, tal acuerdo enseguida no fue respetado. Además, las visitas de familiares y amigos de las nodrizas dentro de la CMEB estaban muy controladas: eran sólo de media hora y previo permiso de la Superiora. A partir de 1890 se ampliaron a una hora dos días a la semana, pero sólo podían acceder parientes próximos ${ }^{56}$. El hecho de que las Hermanas de la Caridad estuvieran a cargo de la Institución hacía que se

\footnotetext{
${ }^{54}$ AHDB, leg. j-3.014, j-3.022 al j-3.028, j-3.044 y j-3.242.

${ }^{55}$ En 1870, a las seis tomaban el desayuno, que era chocolate. Sólo dos horas y media después ingerían el almuerzo, que consistía en sopa y carne, pescado o huevos. A las doce tenían la comida, que se componía de sopa de arroz o pastas, verdura o legumbres y carne. La merienda era también fuerte: ensalada y embutidos, o carne, o huevos, o pescado fresco o escabeche. La cena era sopa de verdura y carne asada. Faltaba en esta dieta la fruta, igual que en la de los expósitos. AHDB, leg. j-3.022 y j-3.028.

${ }_{56}$ AHDB, leg. j-3.020. Reglamento especial para las Casas unidas de Maternidad y Expósitos de la ciudad de Barcelona. Barcelona, 1853, art. 24. Reglamento de la Casa Provincial de Maternidad y Expósitos de Barcelona. Barcelona, 1890, art. 75.
} 
viviera allí un ambiente plenamente religioso y conventual, tal como ocurría en otras inclusas españolas ${ }^{57}$, y como apunta la doctora Valverde (Valverde, 1994: 162-3).

Otro obstáculo en la contratación de amas internas con el que se encontraba la CMEB era que este trabajo exigía una dedicación total, pues estas mujeres tenían que amamantar cinco veces al día e, incluso, a partir de 1870 seis, una de ellas en la madrugada. Además, tenían que lavar las ropas propias y las de los niños, ocuparse de la limpieza de sus salas y colaborar en la elaboración de las comidas, todo ello bajo la dirección e inspección de las Hermanas y de la Junta de Damas de la Institución. Esta vida tan reglamentada llevaba aparejada el castigo en caso de incumplimiento de las normas. Se sancionaba, y en caso de reincidencia se despedía a las negligentes en el cuidado de los niños o en su aseo, a las promotoras de riñas y a las que faltaran al respeto a las Hermanas, a las componentes de la Junta de Damas o a los miembros de la Junta de Gobierno de la $\mathrm{CMEB}^{58}$.

Aparte de dedicación total, las nodrizas internas de la Institución, al igual que las de otras inclusas españolas (Gutiérrez, 1990: 188; Aguirre, 1885: 132-5), se encontraban con que tenían que lactar a dos, tres, e incluso cuatro asilados, contraviniendo el propio reglamento del establecimiento benéfico ${ }^{59}$. Sabemos que en agosto de 1854 veintitrés amas internas lactaban a 87 expósitos, es decir, cada una se hacía cargo de 3,8 asilados. En 1858 la media había bajado a 2,6 niños, aunque seguía siendo alta y aumentaba en los meses de verano ${ }^{60}$. Sin embargo, lo que disuadía a muchas mujeres de ser nodrizas en la CMEB era el miedo a ser contagiadas de sífilis y otras enfermedades venéreas por el expósito al que lactasen. También, en caso de

\footnotetext{
${ }^{57}$ Reglamento para el régimen y gobierno del Gran Hospital de Santiago y de la Casa de Expósitos anexa al mismo. A Coruña, 1881, art. 245.

58 La Junta de Damas estaba compuesta por las señoras de las familias más adineradas y prestigiosas de Barcelona. Su misión era supervisar la labor de las nodrizas y el estado de los expósitos en los locales de la CMEB. Sesión de la Junta de Gobierno de la CMEB, 11-3-1854. AHDB, leg. j-2.027. Reglamento especial para las Casas de Maternidad y Expósitos de la ciudad de Barcelona. Barcelona, 1853, art. 24. AHDB,leg. j-3.020.

${ }^{59}$ Reglamento especial para las Casas unidas de Maternidad y Expósitos de la ciudad de Barcelona. Barcelona, 1853, art. 11.

${ }^{60}$ AHDB, leg. j- 3.014 y j-3.024.
} 
epidemia, las amas tenían más probabilidades de ser atacadas por la enfermedad al residir en locales donde había saturación de personas y donde hasta 1894 no existió un espacio para aislar a los infectados ${ }^{61}$. La escasez de nodrizas era tanta que, en la década de los sesenta del siglo XIX, la Institución admitía a todas cuantas mujeres se presentaban sin que supusiera un obstáculo el que fueran solteras o que tuvieran hijos propios lactantes. Sólo era imprescindible la opinión de los facultativos de la CMEB conforme tenían leche buena y abundante ${ }^{62}$.

Algunas inclusas recurrían, si había escasez de amas, a las mujeres que daban a luz en las maternidades provinciales, obligándolas a lactar a su hijo y a uno o dos asilados más (Uribe, 1996: 198), generalmente de manera gratuita ${ }^{63}$. En Barcelona esta medida no se llevó a cabo hasta 1892, que es cuando se creó de forma efectiva la Maternidad provincial. Hasta ese año las embarazadas que quisieran parir secretamente lo hacían en el Hospital de la Santa Cruz, y este centro benéfico no permitía que estas mujeres ejercieran de nodrizas en la CMEB alegando que con ello se produciría un escándalo y se fomentaría el vicio ${ }^{64}$.

\section{Conclusiones}

Las nodrizas eran la piedra angular de las inclusas. Estas mujeres estaban muy mal pagadas y su sueldo no experimentaba mejoras durante largos períodos de tiempo. La mayoría de ellas vivían en zonas rurales. En la CMEB, además, progresivamente fueron ascendiendo los porcentajes de las residentes fuera de la provincia de Barcelona y de Cataluña. La explicación está en que sólo aceptaban lactar expósitos las mujeres que no tenían la salida profesional del servicio doméstico, la industria, el comercio o un

\footnotetext{
${ }^{61}$ AHDB, leg. 713, j-2.028, j-3.022, j-3.024 y j-3027.

${ }^{62}$ AHDB, leg. j-3.020 y j-3.024.

${ }^{63}$ Reglamento para el régimen interior del Real Hospital de Nuestra Señora de Gracia de Zaragoza. Zaragoza, 1861, art. 204.

${ }^{64}$ AHDB, leg. j-3.024.
} 
nodrizaje particular. Además, estas amas residían en zonas tan pobres como para que les resultara atractivo el sueldo de la $\mathrm{CMEB}$, pues era dinero en metálico que entraba en la unidad familiar, aportado por la mujer. De ahí que muchas aceptaran lactar incluseros a pesar del peligro de contagio de enfermedades, sobre todo venéreas.

Había municipios que se especializaban en el nodrizaje de expósitos. Tenían en común que contaban con una conductora de expósitos y que estaban situados en zonas pobres en las que la agricultura no era importante, los ingresos de los varones eran bajos, y el poco dinero recibido a cambio de lactar un expósito era importante.

Aunque las amas externas tenían que presentar el certificado de su alcalde, de su párroco y de su médico conforme reunían todas las condiciones para amamantar a un niño, el hecho de que fuera unida la lactancia a la miseria provocaba que algunas de estas mujeres hicieran todo lo posible para cobrar o no perder el salario de la inclusa de que se tratara, aún a costa de la salud de los propios expósitos. Frente a las conductas irregulares de algunas nodrizas, la CMEB no ejerció una inspección médica directa sobre estas amas y tampoco controló las condiciones de vida que ofrecían a los niños a su cargo. La propia Institución, que tenía de forma crónica escasez de nodrizas, sabía que no podía encontrar nada mejor a cambio de lo que pagaba.

Respecto a las amas internas, las inclusas tenían dificultades para contratar el número suficiente debido a que muchas mujeres rechazaban este trabajo por lo mal pagado que estaba, por el peligro que comportaba de contraer enfermedades venéreas, y por la falta de libertad y el régimen monjil que imperaba en estas instituciones.

Las nodrizas de las inclusas en general, y las de la CMEB en particular, tenían muy mala consideración social. De ellas se decía que eran avariciosas, descuidadas y que, incluso, llegaban a maltratar a los niños a su cargo. Pero se hablaba poco de su miseria, de los salarios tan bajos que cobraban, pagados muchas veces con mucho retraso, y de la gran cantidad de expósitos, algunos débiles y enfermos, que continuaban viviendo con ellas, a pesar de haber cumplido la edad reglamentaria. 


\section{BIBLIOGRAFÍA}

- Alay, M. (2003): Aprenents i minyones. La infància abandonada a la Barcelona del vuit-cents. Barcelona, Centro de Estudios Ignasi Iglesies.

- Álvarez, L.C. (1978): Marginación social y mentalidad en Andalucía Occidental: Expósitos en Sevilla (1613-1910). Sevilla, Junta de Andalucía.

- Casassas, O. (1993): Metges de nens. Cent anys de pediatria a Catalunya. Barcelona, La Magrana.

- Aguilar, R.; Montiel, J. (2004): La Casa de Maternitat i Expòsits. Les Corts. Barcelona. Diputación y Ayuntamiento de Barcelona.

- Estadísticas sociales. Monografía estadística de la clase obrera. Barcelona, 1921, Ayuntamiento de Barcelona.

- Fernández, J.M. (1996): “La Casa Cuna de Mondoñedo”. En Estudios Mindonienses, $\mathrm{n}^{\circ} 12$, pp. 405-432.

- Fraile, G. (2000): Amas de cría. Valladolid, Fundación Joaquín Díaz.

- Fuentes, M.T. (1997): "Ser nodriza en Barcelona: una posibilidad de supervivencia a final del siglo XIX”. En Anuario de Hojas de Warmi, nº 8, pp. 81-93.

- González, B. (1905): Tratado didáctico de enfermedades de los niños. Madrid.

- González, C. (2001): “Las nodrizas en Almería: ¿Oficio o rol biológico?”. En Actas del III Congreso de Historia de Andalucía. Córdoba, Cajasur.

- Gutiérrez, M. (1990): “Crisis social y asistencia pública en el último cuarto del siglo XIX”. En Historia de la acción social pública en España. Beneficencia y previsión. Madrid, Ministerio de Trabajo y Seguridad Social.

- Junceda, E. (1984): Historia del Real Hospicio y Hospital Real de la Ciudad de Oviedo. Oviedo, Instituto de Estudios Asturianos.

- Krause, M. (1990): “La legislación sobre beneficencia en España. De 1875 a los primeros años del siglo XX”. En Historia de la acción social pública en España. Beneficencia y previsión. Madrid, Ministerio de Trabajo y Seguridad Social. 
- Mause, LL. (1982): Historia de la infancia. Madrid, Alianza.

- Morales, A. (2001): Casa Cuna de Ponferrada, 1775-1937. Ponferrada, Instituto de Estudios Bercianos.

- Puy, J. (2002): Pobres orfes i dements. La beneficència a Girona, 1849-1885. Girona, Ayuntamiento de Girona.

- Recolons, Ll. (1974): La població de Catalumya. Distribució territorial i evolució demogràfica (1900-1970). Barcelona, Laia.

- Rodríguez, A.M. (2003): “La Inclusa de Pontevedra (2872-1903)”. En Pontevedra. Revista de Estudios Provinciais, n ${ }^{\circ}$ 19, pp. 179-204.

- Rodríguez, A.M. (2005): Las mujeres y la beneficencia en la segunda mitad del siglo XIX. La Casa de Maternidad y Expósitos de Barcelona, 1853-1903. Tesis Doctoral. Universidad de Barcelona.

- Rollet, C. (1982): "Nourrices et nourrissons dans le département de la Seine et en France de 1880 à 1940”. En Population, nº 3, pp. 573-604.

- Sarasúa, C. (1994): Criados, nodrizas y amos. El servicio doméstico en la formación del mercado de trabajo madrileño, 1758-1868. Madrid, siglo XXI.

- Soler, E. (2005): El parentesco de leche: la nodriza pasiega en la España de 18301940. Tesis Doctoral. Universidad de Barcelona.

- Tatjer, M. y López, P. (1995): "Evolució demogràfica”. En J. Sobrequés (dir.): Història de Barcelona. Vol. II. Barcelona, Enciclopedia Catalana, pp. 71-122.

- Uribe, A. (1996): Marginalidad protegida: mujeres y niños abandonados en Navarra, 1890-1930. Bilbao, Universidad del País Vasco.

- Valverde, L. (1994): Entre el deshonor y la miseria. Infancia abandonada en Guipúzcoa y Navarra. Siglos XVIII y XIX. Bilbao, Universidad del País Vasco.

- Vega, E. (1992): La mujer en la historia. Madrid, Anaya.

- Vidal, F. y B. (1999): De princesas, señoras y otras clases de mujeres. Madrid, UNED. 


\section{FUENTES}

\section{MANUSCRITAS:}

- Archivo Histórico de la Diputación de Barcelona.

- Sección de Beneficencia. Legajos j-2.027, j-2.028, j-2.206, j-2.298, j-2.394, j-2.395, j2.401, j-2.420, j-2.424, j-2.431, j-2.434, j-3.011, j-3.014, j-3.017, j-3.018, j-3.020, j3.022 al j-3.024, j-3.027 al j-3.030, j-3.038, j-3.062 al j-3.112, j-3.220, j-3.231 al j3.234. Legajos 763, 1.350 al 1.352 y C.C./VII 610

\section{IMPRESAS:}

- Aguirre, J. (1885): Mortalidad en la primera infancia, sus causas y medios de atenuarlas. Madrid.

- Arenal, C. (1861): La beneficencia, la filantropía y la caridad. Madrid.

- Bodas de Oro de la Casa de Maternidad y Expósitos de Barcelona. Barcelona, 1903.

- Censo de la población de España según el empadronamiento verificado el 31-121887. Madrid, 1892.

- Guerra, J. (1887): Higiene de la alimentación de los niños. Barcelona.

- Novella, M. (1882): “Causas de la excesiva mortalidad en la primera infancia en las grandes ciudades y medios de atenuarlas”. En Actas del Congreso Médico Internacional de Sevilla. Sevilla, pp. 301-315.

- Reglamento de la Casa Hospicio y Niños Expósitos de la Provincia de Burgos. Burgos, 1863.

- Reglamento de la Casa de Maternidad y Espósitos de la Provincia de Lugo. Lugo, 1863.

- Reglamento de la Casa Provincial de Maternidad y Expósitos de Barcelona. Barcelona, 1890.

- Reglamento especial para las Casas unidas de Maternidad y Expósitos de la ciudad de Barcelona. Barcelona, 1853. 
- Reglamento para el régimen interior administrativo y económico del establecimiento provincial de Espósitos de Málaga. Málaga, 1856.

- Reglamento para el régimen interior del Real Hospital de Nuestra Señora de Gracia de Zaragoza. Zaragoza, 1861.

- Reglamento para el régimen y gobierno del Gran Hospital de Santiago y de la Casa de Expósitos anexa al mismo. La Coruña, 1881.

- Rull, J. y Cabot, J. (1889): Estudio demográfico-médico del quinquenio de 1883 a 1887 de la Casa Provincial de Maternidad y Expósitos de Barcelona. Barcelona.

DIARIOS Y REVISTAS:

- Artículo sin título. El Diluvio, Barcelona, n 304, 30-10-1896, pp. 5-6.

- “Expósitos y enfermos”. En Revista de Beneficencia, n 4, 15-9-1887.

- "Lactancia mercenaria”. En Revista de Beneficencia, no 18, 22-12-1887, p. 3.

- "Miscelánea". En Revista de Beneficencia, nº 16, 8-12-1887.

- "Real Casa de Beneficencia y Misericordia de La Habana". En Revista de Beneficencia, $\mathrm{n}^{\circ}$ 20, 8-1-1888, pp. 3-4. 